\section{Silicosis, tuberculosis time bomb?}

\section{Dear Editor,}

There is no official data on silica exposure in Portugal, although silicosis is one of the most frequently reported work-related diseases. Most silica exposure in Portugal occurs as a granite quarrying characteristic in the northern region. ${ }^{1}$ Silicotic patients have 2.8-39 times greater risk of developing pulmonary tuberculosis and a 40 times higher risk of dying of tuberculosis than healthy subjects. ${ }^{2,3}$

In order to identify risk factors and clinical characteristics of silico-tuberculosis in Portugal, we analysed all patients notified with tuberculosis between 1999 and 2012. They were classified into two groups - with silicosis (silicoTB) and without silicosis (non-silicoTB). Univariate analysis comprised standard statistical methods: chi-square or Fisher test for the study of independence between two factors, and $t$-test for the assessment of statistically significant differences between means in two independent populations. The classification trees were used to compensate for the fact that some data was missing. These predictive models can determine a smaller subset of predictors, reducing the number of incomplete cases and enabling the posterior use of a different classification method, such as logistic regression. Data were also heavily unbalanced: 205 silicoTB against 16,376 non-silicoTB. A straightforward application of a classification tree would lead to the tree merely composed of the root node, predicting all individuals as non-silicoTB. However, it is known ${ }^{4}$ that resampling the datasets until they are balanced will enhance the performance of the predictive model, and trees in particular. Therefore, random oversampling of the silicosis class until both classes had the same number of observations was performed, and for each oversample, a tree was created. Since the number of silicotics was very small, the resampling methodology provided trees with the same structure and only differing among them in the respective leaves. The tree obtained from the repetition of the silicotic class until exact balance was achieved was therefore considered. Finally, a logistic regression model, using the variables assigned with a predictive importance greater than zero as predictors, was performed. This new dataset consisted of 164 silicotics and 10,432 non-silicotics, all being complete observations. The unbalance was now dealt with in the following way: (1) oversample of the silicosis class until both classes were equally represented; (2) full-size random selection with replacement; (3) logistic regression with the obtained dataset and saving of the estimated coefficients; (4) repetition of the procedure 1000 times. While the first step ensured a balanced dataset, the others incorporated randomness within the silicotic class. A regression coefficient was statistically significant if its values, ignoring the adequate lower and upper percentiles, did not include zero. All statistical analyses were carried out using the $\mathrm{R}$ language and software environment for statistical computation, version 2.12.1. The significance level was set at 0.05 .

Ninety-nine percent of the silicoTB group was male with a mean age of 59.54 years-old (Tables 1 and 2). They all lived in northern Portugal. SilicoTB patients were more prone to be male $(p<0.001)$, older $(p<0.001)$ and have pulmonary disease $(p<0.001)$. Treatment was longer and death was more frequent among silicoTB $(p<0.001)$. In multiple analyses, male sex, age and pulmonary/pleural TB were significant risk factors for silicoTB. Human-immunodeficiency-virus infection (HIV), alcohol and drug abuse were negatively associated with the disease. SilicoTB had more often smear negative and positive culture sputum.

As in other countries, exposure to silica is mostly related to granite quarrying and mining ${ }^{5,6}$ which are activities mostly performed by men but also among ceramic workers (both men and women) - $99 \%$ of the silico-tuberculosis population in the country is male. The older age in this group can be explained by the time needed from exposure to silica dust until silicosis development ${ }^{2,3}$ or by the inclusion of older people (working or retired people, excluding children).

There was a high association of silicosis with pulmonary/pleural TB probably due to its respiratory

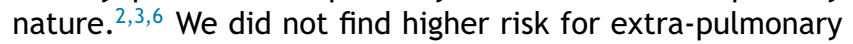
TB, unlike other reports, ${ }^{3}$ which have described a 3.7 times increased risk of extra-pulmonary TB among this group.

Treatment duration is longer in silicoTB group according to treatment guidelines which suggest a 3-month longer period for these patients - in line with the available literature. $^{3}$ Death was more frequent in silicoTB patients - as we do not have access to the cause of death (we just know they died during TB treatment) we cannot fully interpret this result. Other studies suggest that silicoTB patients have increased death rate. ${ }^{2}$

HIV infection, alcohol or drug abuse was less frequent among silicoTB patients. The available literature ${ }^{3}$ report different results probably related to poor living conditions, migrant status and high-risk sexual habits. Our silicotic population are native to Portugal, mostly from rural areas, socially integrated, probably less prone to alcohol, drug abuse or high-risk sexual behaviour.

Finally, our results reveal that silicoTB group were more frequently diagnosed based solely on a positive culture, which emphasizes the difficulties in silicoTB diagnosis. 
Table 1 Sample characteristics.

\begin{tabular}{|c|c|c|c|}
\hline & Cases N (\%) & Controls N (\%) & $p$-value \\
\hline \multicolumn{4}{|l|}{ Gender } \\
\hline Male & $202(99)$ & $11,158(68)$ & \multirow[t]{2}{*}{$<0.001$} \\
\hline Female & $3(1)$ & $5218(32)$ & \\
\hline \multicolumn{4}{|l|}{ Drugs or alcohol } \\
\hline Yes & $159(78)$ & $11,536(70)$ & \multirow[t]{3}{*}{0.090} \\
\hline No & $32(16)$ & $3277(20)$ & \\
\hline Missing & $14(7)$ & $1563(10)$ & \\
\hline \multicolumn{4}{|l|}{ Age (years) } \\
\hline Mean & 59.54 & 43.32 & \multirow[t]{2}{*}{$<0.001$} \\
\hline Sd & 14.56 & 18.23228 & \\
\hline \multicolumn{4}{|l|}{ Main location } \\
\hline Pulmonary or Pleural & $192(94)$ & $13,744(84)$ & \multirow[t]{2}{*}{$<0.001$} \\
\hline Others & $13(6)$ & $2632(16)$ & \\
\hline \multicolumn{4}{|l|}{ Initial microscopy result } \\
\hline Positive & $113(55)$ & $8508(52)$ & \multirow[t]{3}{*}{0.386} \\
\hline Negative & $73(36)$ & $4765(29)$ & \\
\hline Missing & $19(9)$ & 3103 (19) & \\
\hline \multicolumn{4}{|l|}{ Initial culture result } \\
\hline Positive & $153(55)$ & $9910(52)$ & \multirow[t]{3}{*}{0.413} \\
\hline Negative & $25(36)$ & $1977(29)$ & \\
\hline Missing & $27(13)$ & $4489(27)$ & \\
\hline \multicolumn{4}{|l|}{$R X$ thorax } \\
\hline Cavitated & $77(38)$ & $5374(33)$ & \multirow[t]{3}{*}{0.340} \\
\hline Non cavitated & $113(56)$ & $9200(56)$ & \\
\hline Missing & $15(7)$ & $1802(11)$ & \\
\hline \multicolumn{4}{|l|}{ Treatment duration } \\
\hline Lower 1 year & $54(26)$ & $5453(33)$ & \multirow[t]{3}{*}{0.037} \\
\hline Higher 1 year & $149(73)$ & $10,669(65)$ & \\
\hline Missing & $2(1)$ & $254(2)$ & \\
\hline \multicolumn{4}{|l|}{ Drugs resistance } \\
\hline Any resistance & $19(9)$ & $112(1)$ & \multirow[t]{3}{*}{0.340} \\
\hline No resistance & $57(28)$ & $245(1)$ & \\
\hline Missing & $129(63)$ & $16,019(98)$ & \\
\hline \multicolumn{4}{|c|}{ Number of previous treatments } \\
\hline 0 & $176(86)$ & $14,767(90)$ & \multirow[t]{2}{*}{0.052} \\
\hline Higher than 0 & $29(14)$ & $1609(10)$ & \\
\hline \multicolumn{4}{|l|}{ Serology } \\
\hline Positive & $2(1)$ & $1704(10)$ & \multirow[t]{3}{*}{$<0.001$} \\
\hline Negative & $139(68)$ & $9346(57)$ & \\
\hline Unknown & $64(31)$ & $5326(33)$ & \\
\hline Reason end treat & & & \\
\hline Death & $25(12)$ & $1026(6)$ & $<0.001$ \\
\hline Completed treatment & $169(82)$ & $13,974(85)$ & \\
\hline Others & $9(4)$ & $951(6)$ & \\
\hline Missing & $2(1)$ & $425(3)$ & \\
\hline Time between symptoms & & & \\
\hline Lower 1 month & $101(49)$ & 7969 (49) & 0.845 \\
\hline Higher 1 month & $80(39)$ & $6574(40)$ & \\
\hline Missing & $24(12)$ & $1833(11)$ & \\
\hline
\end{tabular}


Table 2 Results obtained after the implementation of the logistic regression model.

\begin{tabular}{|c|c|c|c|c|}
\hline Variable & Estimate & Std. error & LowerBound & UpperBound \\
\hline Constant & -7.850 & 0.261 & 8.337 & -7.357 \\
\hline Age & 0.053 & 0.001 & 0.050 & 0.055 \\
\hline \multicolumn{5}{|l|}{ Main location } \\
\hline Others vs Pulmonary/pleural & -0.867 & 0.105 & -1.086 & -0.673 \\
\hline \multicolumn{5}{|l|}{ HIV serology } \\
\hline Negative vs positive & 2.074 & 0.202 & 1.692 & 2.493 \\
\hline Unknown vs positive & 1.784 & 0.205 & 1.391 & 2.204 \\
\hline \multicolumn{5}{|l|}{ Gender } \\
\hline Male vs female & 3.710 & 0.130 & 3.477 & 3.969 \\
\hline \multicolumn{5}{|l|}{ Drugs or alcohol } \\
\hline Yes vs No & -0.482 & 0.049 & -0.577 & -0.387 \\
\hline \multicolumn{5}{|l|}{ Initial microscopy } \\
\hline Positive vs negative & -0.300 & 0.045 & -0.389 & -0.210 \\
\hline \multicolumn{5}{|l|}{ Initial culture } \\
\hline Positive vs negative & 0.187 & 0.059 & 0.068 & 0.303 \\
\hline
\end{tabular}

\section{Conflicts of interest}

The authors have no conflicts of interest to declare.

\section{Acknowledgements}

A. Rita Gaio is partially supported by CMUP (UID/MAT/ 00144/2013), which is funded by FCT (Portugal) through the programs FEDER, under the partnership agreement PT2020.

\section{References}

1. Centro nacional de proteção contra riscos profissionais. Lisboa: Relatório anual; 2005.

2. Calvert G. Occupational silica exposure and risk of various diseases: an analysis using death certificates from 27 states of the United States. Occup Environ Med. 2003;60:122-9.

3. Hnizdo E, Murray J. Risk of pulmonary tuberculosis relative to silicosis and exposure to silica dust in South African gold miners published erratum appears in Occup Environ Med 1999 Mar;56(3):215-6. Occup Environ Med. 1998;55:496-502.

4. R: a language and environment for statistical computing. Vienna: R Development Core Team; 2011.

5. The National Institute for Occupational Safety and Health. Health effects of occupational exposure to respirable crystalline silica; 2002.

6. Pérez-Alonso A, Cordoba J, Millares-Lorenzo J, Figueroa-Murillo E, Garcia-Vadillo C, Romero-Morillo J. Outbreak of silicosis in Spanish quartz conglomerate workers. Int J Occup Environ Health. 2014;20:26-32.

V. Melo ${ }^{a}$ L. Baía ${ }^{b}$, A. Rita Gaio ${ }^{b, c}$, R. Duarte ${ }^{d, e, f, g, *}$

a Pulmonology Department, Centro Hospitalar

Tondela-Viseu, Portugal

b Faculty of Sciences, Department of Mathematics, University of Porto, Portugal

c Centre for Mathematics of the University of Porto, Portugal

dEPIUnit Institute of Public Health, University of Porto, Portugal

e Chest Disease Centre Vila Nova de Gaia, Portugal

f Pulmonology, Centro Hospitalar de Vila Nova de Gaia/Espinho EPE, Portugal

' Department of Clinical Epidemiology, Predictive Medicine and Public Health, University of Porto Medical School, Portugal

* Corresponding author.

E-mail address: raquelafduarte@gmail.com (R. Duarte).

Available online 04 September 2016

http://dx.doi.org/10.1016/j.rppnen.2016.07.005

2173-5115/

(c) 2016 Sociedade Portuguesa de Pneumologia. Published by Elsevier España, S.L.U. This is an open access article under the CC BY-NC-ND license (http://creativecommons.org/licenses/by-nc-nd/4.0/). 